\title{
DIVULGAÇÃO DA CIÊNCIA E CONSERVAÇÃO DOS RECIFES DE CORAL DA PARAÍBA,
} ATLÂNTICO SUL OCIDENTAL

Dissemination of science and coral reefs conservation at Paraiba State, South West Atlantic

\section{Christinne Costa Eloy ${ }^{1}{ }^{\circ} ;$ Henrique E. C. França ${ }^{2}$; $;$ Karina Massei $^{3}$}

\section{Resumo}

A saúde do oceano reflete diretamente o bem-estar da humanidade. As mudanças globais protagonizadas pelo aumento da população humana já atingem todos os sistemas naturais, colocando em risco o suporte biológico do planeta e impactando sobremaneira os ecossistemas marinhos. Para atender à agenda 2030 e ampliar a cooperação internacional em torno do ODS 14 - conservar e usar de forma sustentável os oceanos-, foi declarada pelas Nações Unidas a Década da Ciência Oceânica (2021-2030). Fazendo um recorte para o Atlântico Sul Ocidental, um dos ecossistemas marinhos mais ameaçados são os recifes de coral do nordeste brasileiro. Caracterizados por pequeno número de espécies, mas forte endemismo, são fundamentais para o equilíbrio ecológico e econômico da região. As piscinas naturais que se formam nos recifes costeiros são intensamente exploradas pela visitação turística e, apesar de contar com duas Unidades de Conservação marinhas protegidas por lei, problemas de gestão e fiscalização ameaçam a conservação da biodiversidade, além da falta de informações sobre sua importância e vulnerabilidade. Com o objetivo de investigar o processo de comunicação da ciência para o público em geral, foi realizado um levantamento de textos de divulgação relacionados ao tema no período de 2018 a 2020 - a partir da plataforma Google Notícias -, para identificar quantos e quais deles abordavam questões relacionadas aos recifes de coral da Paraíba, além da percepção da presença de especialistas dando voz a esses conteúdos. Os resultados apontam para a incidência quase nula da divulgação a respeito desses ambientes, à medida em que esse ecossistema tem enfrentado processo de degradação praticamente desconhecido da sociedade em geral, o que pode refletir nas condutas inadequadas encontradas em alguns visitantes. Fica um alerta para a necessidade de ampliar a divulgação das investigações desenvolvidas pelos institutos de pesquisa e universidades sobre os ecossistemas de recifes de coral da região, somando esforços ao Plano de Comunicação e Engajamento construído para a Década do Oceano. A divulgação da ciência é, sem dúvida, uma ferramenta essencial para o envolvimento da sociedade em prol de um oceano saudável.

Palavras-chave: Década do Oceano. Divulgação da Ciência. Recifes de Coral. Conservação. Comunicação da Ciência.
1 Doutoranda em Divulgação da pela Universidade do Porto, Portugal. Professora de Biologia no Instituto Federal de Educação, Ciência e Tecnologia da Paraíba, Brasil.

*christinne.eloy@ifpb.edu.br

2 Doutorando em Ciência da informação - Universidade de Coimbra, Portugal. Professor em Arquivologia- Universidade Estadual da Paraíba, Brasil.

*franca.henrique@gmail.com

3 Pós-doutoranda no Programa de Ecologia e Monitoramento Ambiental da Universidade Federal da Paraíba (PPGEMA/UFPB). Doutora em Ciências Ambientais (PRODEMA/UFPB). Presidente do InPact - Instituto de Pesquisa e Ação

*karina.massei@inpact.org.br 


\section{Abstract}

The health of the ocean directly reflects the well-being of humanity. Global changes caused by the increase in human population are already affecting all natural systems, endangering the biological support of the planet and greatly impacting marine ecosystems. To meet the 2030 agenda and expand international cooperation around SDG 14 - to conserve and sustainably use the oceans - the United Nations has declared a Decade of Ocean Science (2021-2030). Cutting across the Western South Atlantic, one of the most threatened marine ecosystems are the coral reefs of northeastern Brazil. Characterized by a small number of species, but strong endemism, they are fundamental to the ecological and economic balance of the region. The natural pools that are formed in the coastal reefs are intensively explored by tourist visitation and, despite having two Marine Conservation Units protected by law, management and inspection problems threaten the biodiversity conservation, besides the lack of information on its importance and vulnerability. Aiming to investigate the process of science communication to the general public, a survey of dissemination texts related to the theme in the period 2018 to 2020 - from the Google News platform - was conducted to identify how many and which of them addressed issues related to the coral reefs of Paraiba, in addition to the perception of the presence of experts giving voice to these contents. The results point to the almost null incidence of disclosure about these environments, to the extent that this ecosystem has faced a degradation process virtually unknown to society in general, which may reflect in the inappropriate behaviors found in some visitors. This is an alert for the need to expand the dissemination of investigations developed by research institutes and universities on coral reef ecosystems in the region, adding efforts to the Communication and Engagement Plan built for the Ocean Decade. The dissemination of science is undoubtedly an essential tool for society's engagement for a healthy ocean.

Key words: Ocean Decade. Science dissemination. Coral Reefs. Conservation. Science Communication. 


\section{Introdução}

$\mathrm{E}$ stamos vivendo uma nova era, o Antropoceno, protagonizada pelas atividades humanas que têm desencadeado profundas mudanças no meio ambiente, desde alterações nos principais ciclos biogeoquímicos da Terra até a evolução da vida (Crutzen \& Stoermer, 2000; Lewis \& Maslin, 2015; Steffen et al., 2011). As evidências de mudanças globais sugerem que cruzamos um limite de escala de época dentro dos últimos dois séculos (Zalasiewicz et al., 2011), mostrando o quão acelerado tem sido esse processo.

O crescimento da população humana mundial que caracteriza o Antropoceno é uma das razões para a magnitude dos impactos dessa era (Crutzen \& Stoermer, 2000; Steffen et al., 2011). O relatório do Painel Intergovernamental sobre Mudanças Climáticas (IPCC) aponta que, desde os períodos préindustriais, tem-se registrado um aumento dramático na concentração de dióxido de carbono $\left(\mathrm{CO}_{2}\right)$ atmosférico (IPCC, 2014) e pode-se destacar esse fator como responsável por alterações importantes, como o aumento da temperatura global, que já se reflete em todos os sistemas naturais e têm sido particularmente impactante sobre os ecossistemas marinhos (Tyrrell, 2011).

A acidificação do oceano é uma consequência inevitável do aumento desse gás na atmosfera (Lenton et al., 2018), resultando na alteração dos ciclos biológicos dos organismos que produzem $\mathrm{CaCO}_{3}$, incluindo algas calcárias e corais de águas rasas (Biru, et al., 2020; Orr et al., 2005; Tyrrell, 2011).

Com a pressão das alterações climáticas sobre a biosfera, a comunidade internacional aprovou, em 2015, a Agenda 2030 para o Desenvolvimento Sustentável - um programa que resultou do trabalho conjunto de governos e cidadãos de todo o mundo para criar um novo modelo global de desenvolvimento, incorporando as dimensões econômica, social e ambiental (Nações Unidas, 2015).

O texto das Nações Unidas (2015) traz os Objetivos do Desenvolvimento Sustentável (ODS) e reafirma que a mudança climática é um dos maiores desafios do nosso tempo e enfatiza que o aumento na temperatura global e a acidificação dos oceanos não afetam apenas as áreas costeiras, mas coloca em risco a sobrevivência de muitas sociedades e dos sistemas de suporte biológico do planeta.

Entre os dezessete ODS, destacamos o objetivo de número catorze "conservar e usar de forma sustentável os oceanos, mares e recursos marinhos para o desenvolvimento sustentável"1. Para ampliar a cooperação internacional em torno do ODS 14 , as Nações Unidas declararam os anos de 2021 a 2030 a Década da Ciência Oceânica para o desenvolvimento sustentável, um esforço conjunto na tentativa de sensibilizar o mundo para a relevância desse ecossistema e sobre as ameaças a que estão submetidos (Organização das Nações Unidas [ONU], 2017).

Fazendo um recorte para o Atlântico Sul Ocidental, um dos ecossistemas marinhos mais ameaçados são os recifes costeiros. Estendem-se por três mil quilômetros e possuem características próprias que os distinguem de outros recifes de coral do mundo pois, apesar da pequena diversidade de espécies, exibem um alto grau de endemismo (Leão et al, 2010; 2003), o que reforça sua relevância para o equilíbrio ecológico e econômico da região.

A região costeira da Paraíba (Figura 1) apresenta ecossistemas recifais que abrigam espécies ameaçadas e endêmicas do Brasil (Figura 2) (Leão et al., 2010; Gondim et al., 2011), recebendo anualmente inúmeros visitantes do Brasil e de outros países, com o agravante da falta de gestão adequada desse turismo na região (Debeus \& Crispim, 2008; Melo et al., 2014; Souza et al., 2016).

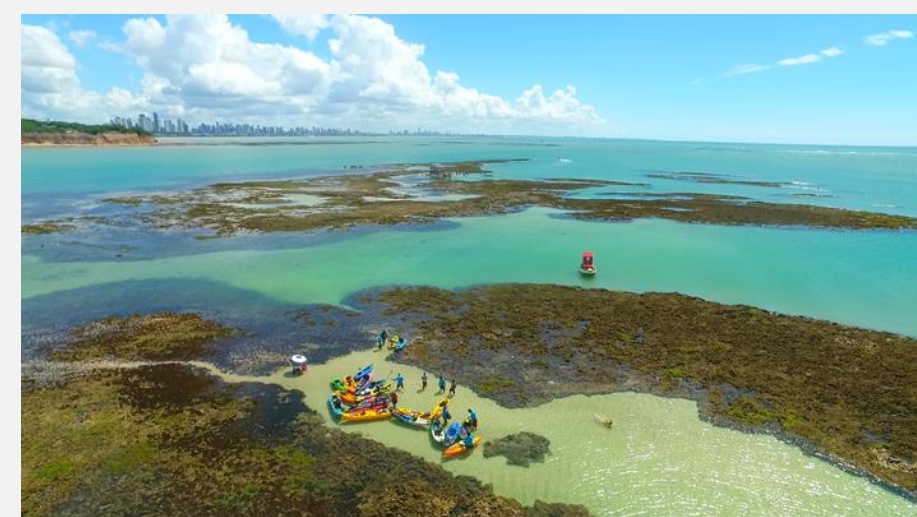

Figura 1. Recifes costeiros da Paraíba (Massei, 2018).

\footnotetext{
${ }^{1}$ https: / / nacoesunidas.org/pos2015/agenda2030/
} 


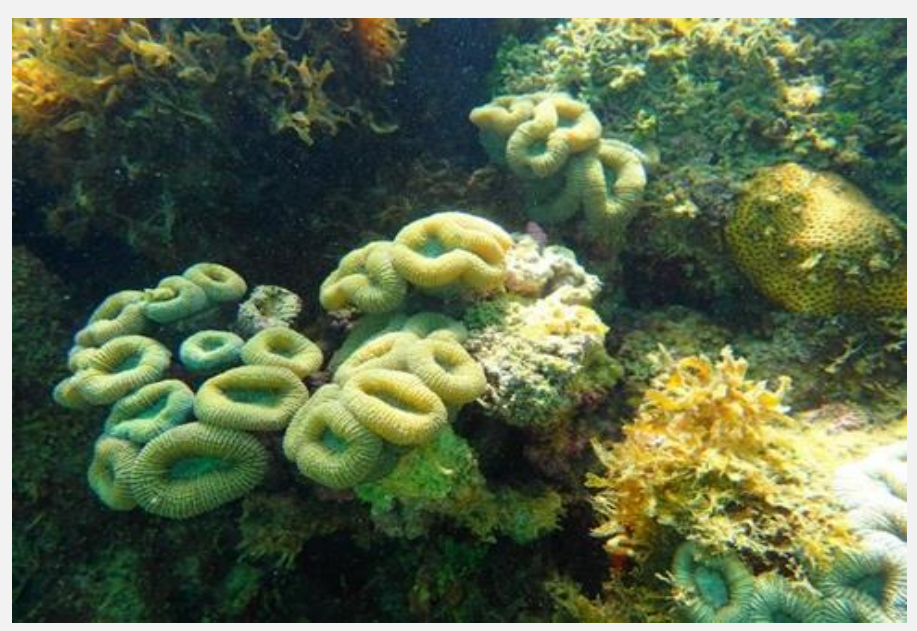

Figura 2. Espécime de coral Mussismilia hartti (Verril, 1867), endêmico do Brasil, está listado como em perigo na lista vermelhas de espécies ameaçadas. Acervo pessoal.

O litoral paraibano abriga duas Unidades de Conservação (UCs) marinhas - uma criada em 2000, - Parque Estadual Marinho de Areia Vermelha $(\mathrm{PEMAV})^{2}$ e a mais recente, criada em 2018, a Área de Proteção Ambiental do Naufrágio Queimado ${ }^{3}$ (APANQ). Embora protegidas por lei, muitos dos visitantes desconhecem essa condição realizando, por vezes, atividades expressamente proibidas, como caminhar sobre o recife e coletar material biológico para souvenier (Santos et al., 2013). Várias pesquisas realizadas na área já apontaram para problemas de gestão, como falta de fiscalização adequada e implementação de plano de manejo, além de divulgação de informações imprecisas/equivocadas (Costa et al., 2007; Gondim et al., 2011; Lourenço et al., 2015; França e Eloy, 2017).

Os ecossistemas costeiros mais visitados na Paraíba são o PEMAV e os recifes de Picãozinho, Seixas e Penha. Após anos de exploração turística sem ordenamento, Picãozinho passou a ter o número de visitantes limitado como resultado de estudo de capacidade de carga desenvolvido por pesquisadores (Debeus et al., 2008; 2012), configurando como um dos poucos locais com recifes de coral na região com ordenamento para o turismo. Nas piscinas naturais do Seixas, a visitação até 2005 era realizada por pescadores e pequenas embarcações, com pesca

\footnotetext{
2 Decreto Estadual $n^{\circ} 21.263,07$ de fevereiro de 2000. Unidade de Proteção Integral Parque Estadual Marinho de Areia Vermelha - SUDEMA/Paraíba.
}

artesanal, lazer e turismo como principais usos (Melo et al., 2014).

Atualmente, recebe entre 10 e 12 embarcações que transportam mais de 100 visitantes, cada, em um único dia. No entanto, a população local desconhece a maior parte de sua diversidade biológica, seja por falta de oportunidade de visitar os recifes, seja por falta de acesso a informações relevantes ou a facilidade de acesso a informações equivocadas ou superficiais sobre eles. Essas evidências apontam para a precariedade de atenção que esses ecossistemas têm recebido por parte de gestores e sociedade em geral.

Por mais contraditório que pareça, o aumento do conhecimento produzido sobre a importância do oceano para a saúde e equilíbrio do planeta tem sido diretamente proporcional ao aumento das pressões das atividades humanas sobre esse ecossistema (Lotze et al., 2018). Sabe-se que a mídia em geral tem um papel fundamental na formação de opinião pública influenciando, inclusive, a agenda política. Nesse cenário, a interação entre ciência e sociedade torna-se crucial, sendo necessário ampliar o acesso do público em geral a informações de caráter científico e ir além, gerando envolvimento necessário para mudanças de atitude frente às demandas para a conservação.

Na chamada Sociedade da Informação é notório que a transformação se dá, invariavelmente, pela via da comunicação. Se os dados científicos são sólidos, faz-se fundamental comunicá-los de forma clara e ampla, para que essa informação possa gerar mudanças sociais, a partir dos indivíduos a quem se comunica - desde aqueles que interagem diretamente com os ecossistemas marinhos (pescadores, guias de turismo, gestores, etc) àqueles que sequer tiveram contato, mas são afetados indiretamente por eles.

Magrani (2014) nos aponta que, com a mudança do perfil da sociedade moderna - sociedade essa hiperconectada -, as formas do comunicar, de divulgar dados, são um potencializador de engajamento social. Isso também vale, evidentemente, para a ciência. Afinal, não é de hoje que a comunidade científica (e seus entusiastas) tem

3 Decreto Estadual $n^{\circ}$ 38.931, 28 de dezembro de 2018. Área de Proteção Ambiental do Naufrágio Queimado SUDEMA/Paraíba. 
utilizado caminhos para chegar ao público em geral, não especializado, lançando mão das tecnologias de comunicação disponíveis a seu tempo (Bucchi e Trench, 2008). Das revistas acadêmicas especializadas às redes sociais, dos congressos científicos às notícias na web, diversos estudiosos têm se debruçado sobre esse ecossistema comunicacional (You, 2014; Bonaiuti, 2015; Ranger e Baltitude, 2016).

Cidadãos, atores da administração pública, empresários, terceiro setor, grupos da sociedade civil organizada e tomadores de decisão, todos podem e devem ter suas decisões influenciadas pela divulgação da ciência, com suas bases de dados e análises consolidadas. Disso depende a própria retomada da influência e credibilidade da ciência junto à sociedade. "Esta nova 'abertura' da ciência ao espaço público poderá relacionar-se com uma tentativa de reforço do seu poder social" (Carvalho, 2004, p.46).

Com o objetivo de investigar esse processo comunicacional, analisamos a ocorrência e estratégias de divulgação de informações sobre os recifes costeiros do estado para o público em geral, ou seja, nos meios de comunicação em massa.

\section{MATERIAL E MÉTODOS}

Foi utilizado material de divulgação produzido para mídia eletrônica, incluindo apenas os materiais de acesso gratuito. 0 foco em material de divulgação disponível online se justifica pela facilidade de acesso e, principalmente, por conseguir englobar tanto grandes veículos de comunicação quanto a mídia local.

A partir da plataforma Google Notícias, foi realizado o levantamento de textos de divulgação relacionados a recifes de coral publicados no período de três anos (2018-2020), para identificar quantos e quais deles abordavam questões relacionadas aos recifes de coral da Paraíba, além da percepção da presença de especialistas/investigadores dando voz a esses conteúdos.

Foram realizadas 3 buscas com as seguintes palavraschave: "corais da Paraíba", "recifes costeiros da Paraíba", "recifes costeiros".

\section{RESULTADOS E DISCUSSÃO}

A busca por "corais da Paraíba" resultou em quatro matérias, sendo três delas sobre o mesmo conteúdo, relacionado com 0 evento de branqueamento ocorrido no verão de 2019/2020 e registrado em maio deste último ano, sendo divulgado por pesquisadores da Universidade Federal da Paraíba (Figura 3). A matéria foi veiculada em um portal de notícias local no dia dezenove de maio e, em agosto e dezembro, respectivamente, foi publicada no portal online da revista Galileu e no Hypeness - este último apenas replicou a mesma matéria publicada na Galileu (ver Tabela 1).

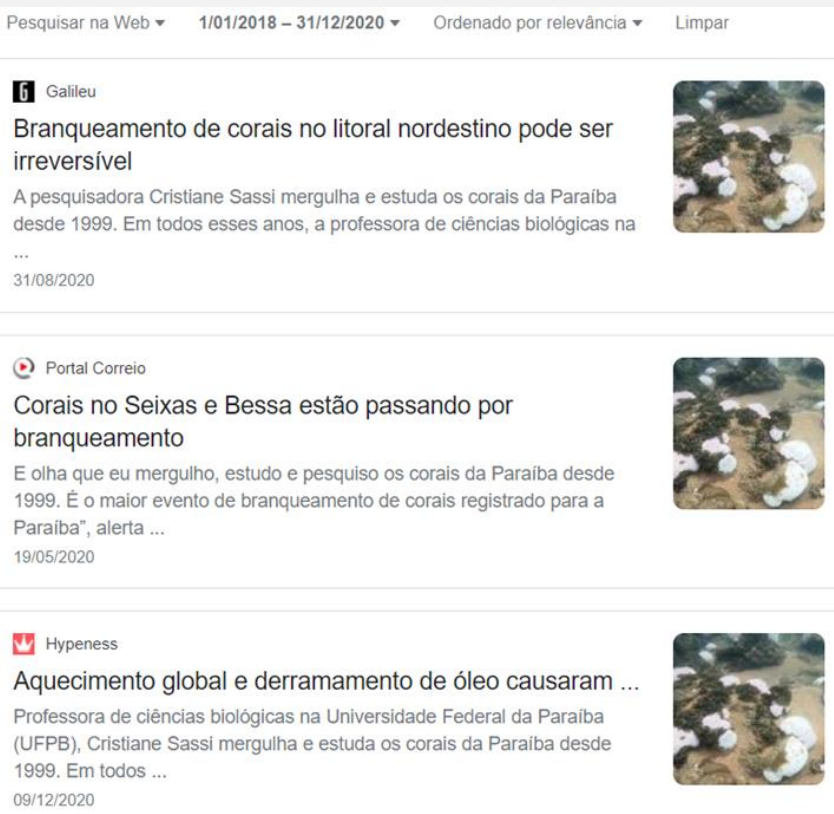

Figura 3. Resultado encontrado no Google Notícias para a busca "corais da Paraíba".

Nas três matérias há informações disponibilizadas de participação direta do pesquisador. A quarta notícia encontrada na busca tratava-se de um artigo de opinião e foi deixada de fora da tabela.

\begin{tabular}{|l|c|c|c|}
\hline \multicolumn{1}{|c|}{ Título da Matéria } & Mídia & Data & $\begin{array}{c}\text { Participação de } \\
\text { Pesquisador }\end{array}$ \\
\hline $\begin{array}{l}\text { Branqueamento de corais no litoral } \\
\text { nordestino pode ser irreversível }\end{array}$ & Revista Galileu & $31 / 08 / 2020$ & Sim \\
\hline $\begin{array}{l}\text { Corais no Seixas e Bessa estão passando por } \\
\text { branqueamento }\end{array}$ & Portal Correio & $19 / 05 / 2020$ & Sim \\
\hline $\begin{array}{l}\text { Aquecimento global e derramamento de óleo } \\
\text { causaram branqueamento de corais no } \\
\text { Nordeste }\end{array}$ & Hypeness & $09 / 12 / 2020$ & Sim \\
\hline
\end{tabular}


Tabela 1. Notícias relacionadas aos recifes de coral da Paraíba encontradas no "Google Notícias", na busca por "corais da Paraíba", entre 2018 e 2020.

A busca pela palavra-chave "recifes costeiros da Paraíba" resultou em apenas uma notícia (Figura 4), coincidindo com a veiculada no dia 19 de maio de 2020, pelo portal do Jornal Correio, já detectado na pesquisa anterior. 0 termo "recifes costeiros" foi considerado na pesquisa por ser um termo utilizado com frequência para referir os recifes de coral do Nordeste brasileiro.

Essa referência se destaca quando é feita a pesquisa apenas no termo "recifes costeiros", sem dar destaque à Paraíba. 0 resultado da busca para esse termo ("recifes costeiros") resgatou 34 notícias. Destas, apenas três foram relacionadas aos recifes de coral da Paraíba (Tabela 2). Novamente aparece a notícia do Portal Correio sobre o branqueamento e aparecem duas novas matérias, ambas sobre 0 mesmo tema, mas veiculadas em mídias diferentes. Essas notícias falavam sobre a criação da Área de Proteção Ambiental do Naufrágio Queimado, sendo uma veiculada no dia nove de julho de 2019 pelo Portal Correio, com destaque para a proteção dos naufrágios com a criação da APA, e a outra no site da EBC (Empresa Brasil de Comunicação), com destaque para o aumento do percentual de áreas de preservação costeira na Paraíba.

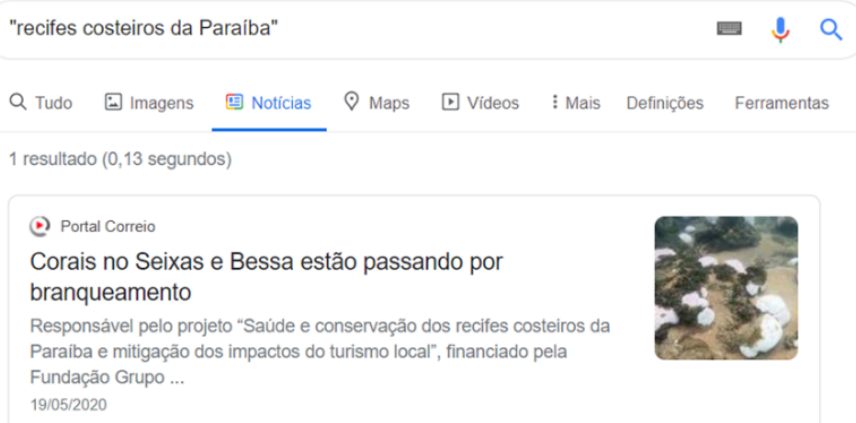

Figura 4. Resultado encontrado no Google Notícias para a busca "recifes costeiros da Paraíba".

As 31 matérias restantes eram relativas a outras localidades no Brasil e fora dele, principalmente sobre a barreira de corais da Austrália, e outras relativas a temas diversos que não os recifes de coral.
Tabela 2. Notícias encontradas no "Google Notícias", na busca por "recifes costeiros" nos últimos 3 anos, destacando apenas as relativas ao Estado da Paraíba.

\begin{tabular}{|l|c|c|c|}
\hline \multicolumn{1}{|c|}{ Título da Matéria } & Media & Data & $\begin{array}{c}\text { Participação } \\
\text { de } \\
\text { Pesquisador }\end{array}$ \\
\hline $\begin{array}{l}\text { Corais no Seixas e Bessa estão } \\
\text { passando por branqueamento }\end{array}$ & Portal Correio & $19 / 05 / 2020$ & Sim \\
\hline $\begin{array}{l}\text { Área de proteção aumenta para } \\
10,7 \% \text { a preservação }\end{array}$ & EBC & $14 / 07 / 2019$ & Sim \\
\hline $\begin{array}{l}\text { Embarcações naufragadas na } \\
\text { Paraíba serão protegidas por lei }\end{array}$ & Portal Correio & $09 / 07 / 2019$ & Sim \\
\hline
\end{tabular}

\section{CONSIDERAÇÕES FINAIS}

Esses resultados demonstram que pouco tem sido feito em relação à divulgação para a sociedade em geral sobre os recifes de coral do Estado da Paraíba, aumentando a vulnerabilidade desse ecossistema tanto às alterações globais quanto aos estresses provenientes de impactos locais.

O bem-estar e o equilíbrio das condições de saúde dos ambientes marinhos reverberam diretamente na qualidade de vida dos seres humanos. Nesse imenso 'universo aquático', a mínima presença (menos de $0,1 \%$ ) dos recifes de coral simboliza proteção (como berçário de espécies), recursos medicinais (como a produção de medicamentos a partir de seus componentes), econômicos (pela pesca e pelo turismo).

Este trabalho ressalta o alerta para a conservação da vida no mar. Na potencial ligação entre dados científicos e engajamento social para a conservação marinha está a divulgação da ciência. Com ênfase nessa premissa, buscou-se averiguar em que medida os recifes de coral da Paraíba têm sido divulgados pela mídia. Apesar da busca não ter sido exaustiva, os resultados mostram divulgação pouco relevante, destoando da exploração das zonas de turismo marinho da costa paraibana.

A ausência quase total de divulgação desses ecossistemas deságua no enfraquecimento dos cuidados para com os recifes pela população e mesmo pelo poder público. No cenário atual em que a desinformação e o desinteresse geral pelas questões ambientais se acentuam no país, promover e permitir o acesso à informação de qualidade, aliada a ferramentas que despertam o interesse do público, é uma estratégia poderosa a favor da conservação de nosso patrimônio natural. 
Este trabalho busca alertar para que os ecossistemas marinho-costeiros da Paraíba recebam o cuidado necessário de todos os atores sociais.

Com vistas ao futuro, enfatizamos a necessidade da divulgação da ciência no contexto e para além dele. Pois este exemplo, embora local, reflete-se globalmente, estendendo-se a outras regiões desse oceano comum. É neste contexto que a Década da Ciência Oceânica para o desenvolvimento sustentável (2021-2030) traz um Plano Global de Implementação, gerido pela Comissão Oceanográfica Intergovernamental da UNESCO (UNESCO-COI), que apresenta o conceito de ciência transformadora, ressaltando a importância do desafio de comunicar a ciência de forma a gerar uma visão participativa no processo científico, alinhada às demandas locais e globais.

Assim, em nível nacional, neste primeiro ano de navegação na década oceânica, o trabalho coletivo realizado em diversas oficinas que integraram 0 saber e fazer das regiões, possibilitando a formação de redes, engajamento e comunicação, resultou na elaboração do Plano de trabalho bianual de Comunicação e Engajamento (MCTI, 2021) alinhado com o Comitê Internacional. Em linguagem acessível e direcionada a cada público específico, ou seja, sem deixar ninguém para trás, a divulgação da ciência oceânica é a chave para a compreensão do que temos e do que é necessário construir para o futuro que queremos - um oceano limpo, saudável, resiliente, previsível, seguro, produtivo, transparente, acessível, valorizado e conhecido por todos (MCTI, 2021). Pois, como pontua Knorr-Cetina (1999), "a ciência projeta-se a si mesma no futuro através da comunicação".

\section{REFERÊNCIAS}

Biru, F. N., Cazzonelli, C. I., Elbaum, R. \& Johnson, S. N. (2020) Contrasting effects of Miocene and Anthropocene levels of atmospheric $\mathrm{CO} 2$ on silicon accumulation in a model grass. Biol. Lett. http://doi.org/10.1098/rsbl.2020.0608

Bonaiuti, G. (2015). Academic Social Networks: How the web is changing our way to make and communicate researches. Research on education and media, 7(2), 3-14.
Bucchi, M., \& Trench, B. (Eds.). (2008). Handbook of public communication of science and technology. Routledge.

Carvalho, A. (2004). Política, cidadania e comunicação "crítica" da ciência. Comunicação e Sociedade, 6 , 35-49. https://doi.org/10.17231/comsoc.6(2004).1227

Costa, C. F., Sassi, R., Costa, M. A. J. \& Brito, A. C. L. (2007) Recifes costeiros da Paraíba, Brasil: usos, impactos e necessidades de manejo no contexto da sustentabilidade. Gaia Scientia 9(1) 37-45.

Crutzen, P. J. \& Stoermer, E. F. (2000). The Anthropocene. IGBP Global Change News. 41, 17-18.

Debeus, G., Crispim M.C. (2008) O Turismo nas piscinas naturais de Picãozinho, João Pessoa, PB Percepções, Conflitos e Alternativas. Revista de estudos ambientais, 10(1):21-32.

Debeus, G., Lima, E. R. V., Crispim, M. C. (2012) Proposta de zoneamento para o ambiente recifal de Picãozinho, João Pessoa - Pb. Gaia Scientia.

França, H. E. C. e Eloy, C. C. (2017) Negligência comunicacional e sensacionalismo ambiental: o caso do Parque Estadual Marinho de Areia Vermelha. Tierra: Naturaleza, Biodiversidad Y Sustentabilidad. Ed. Rigoberto Rodriguez Quirós. 1 ed. San José, Costa Rica.

Gondim, A. I., Dias, T. L. P., Campos, F. F., Alonso, C. \& Christoffersen, M. L. (2011) Macrofauna bêntica do Parque Estadual Marinho de Areia Vermelha, Cabedelo, Paraíba, Brasil. Biota Neotropica 11(2): 1-12.

IPCC. (2014). Climate Change 2014: impacts, adaptation, and vulnerability. Part A: global and sectoral aspects. Contribution of working group II to the fifth assessment report of the intergovernmental panel on climate change. Cambridge University Press.

Knorr-Cetina, K. (1999) Epistemic Cultures: how the sciences make knowledge. Cambridge. Mass: Harvard University Press.

Leão, Z.M.A.N., Kikuchi, R.K.P., Oliveira, M.D.M., Vasconcelos, V. (2010) Status of Eastern Brazilian 
coral reefs in time of climate change. Panam. J. Aquat. Sci., 5(2) 224-235.

Leão, Z.M.A.N., Kikuchi, R.K.P., Testa, V. (2003) Corals and coral reefs of Brazil. In: CORTÉZ, J. (Ed). Lat. Amer. Coral Reefs, Elsevier, 9-55.

Lenton, A., Matear, R. J., Keller, D. P., Scott, V. \& Vaughan, N. E. (2018) Assessing carbon dioxide removal through global and regional ocean alkalinization under high and low emission pathways. Earth Syst. Dynam. 9, 339-357. https://doi.org/10.5194/esd-9-339-2018

Lewis, S. L., and Maslin, M. A. (2015). Defining the Anthropocene. Nature 519, 171-180. doi: $10.1038 /$ nature 14258

Lotze, H. K., Guest, H., O'Leary, J., Tuda, A., Wallace, D. (2018) Public perceptions of marine threats and protection from around the world. Ocean and Coastal Management 152: 14-22. http://doi.org/10.1016/j.ocecoaman.2017.11.004

Lourenço, L. J. S., Eloy, C. C., Crispim, M. C. (2015). Caracterização do Parque Estadual Marinho de Areia Vermelha, Cabedelo- $\mathrm{Pb}$, baseada na diversidade e abundância dos cnidários da classe Anthozoa, como subsídio para o zoneamento ecológico econômico. Gaia Scientia 9(1).

Magrani, E. (2014). Democracia conectada: a internet como ferramenta de engajamento políticodemocrático. Juruá.

MCTI. (2021). Plano Nacional de Implementação da Década da Ciência Oceânica para o Desenvolvimento Sustentável. Coordenação-Geral de Oceano, Antártica e Geociências. Secretaria de Pesquisa e Formação Científica. Ministério da Ciência, Tecnologia e Inovações. CGOA - MCTI. 21 p.

Melo, R. S., Lins, R. P. M. \& Eloy, C. C. (2014). O impacto do turismo em ambientes recifais: caso praia Seixas-Penha, Paraíba, Brasil. Rede, 8(1),67-83.

Nações Unidas. (2015). Transforming our world: The 2030 agenda for sustainable development (resolution adopted by the General Assembly on 25 September 2015.

A/RES/70/1. https: //www.un.org/ga/search/view_doc.asp?symb ol $=\mathrm{A} / \mathrm{RES} / 70 / 1$ \&Lang $=\mathrm{E}$

Organização das Nações Unidas [ONU]. (2017). UN designates 2021 - 2030 'Decade of Ocean Science'. https: / / news.un.org/en/story/2017/12/638452-undesignates-2021-2030-decade-ocean-science.

Orr, J., Fabry, V., Aumont, O. et al. (2005) Anthropogenic ocean acidification over the twentyfirst century and its impact on calcifying organisms. Nature 437, 681-686. https://doi.org/10.1038/nature04095

Ranger, M., \& Bultitude, K. (2016). 'The kind of mildly curious sort of science interested person like me': Science bloggers' practices relating to audience recruitment. Public Understanding of Science, 25(3), 361-378.

Riesch, H., \& Mendel, J. (2014). Science blogging: Networks, boundaries and limitations. Science as Culture, 23(1), 51-72.

Souza, M. C. S., Vianna, P. G. C., Massei, K., Lima, R. C., Eloy, C. C. (2016) Análise espacial e mapeamento da ocorrência de corais nos recifes de Picãozinho, João Pessoa - Pb, comparativo entre 2001 e 2015/2016. Gaia Scientia 10(4): 432-445. doi: $10.21707 / g s . v 10 . n 04 a 34$

Steffen, W., Grinevald, J. Crutzen, P \& McNeill, J. (2011) The Anthropocene: conceptual and historical perspectives. Phil. Trans. R. Soc. A. 369: 842-867. http://doi.org/10.1098/rsta.2010.0327

Tyrrell, T. (2011) Anthropogenic modification of the oceans. Phil. Trans. R. Soc. A. 369: 887-908. http://doi.org/10.1098/rsta.2010.0334

You, J. (2014). Who are the science stars of Twitter?. Recuperado em https://science.sciencemag.org/content/345/6203 /1440.summary

Zalasiewicz, J., Williams, M., Haywood, A. \& Ellis, M. (2011). The Anthropocene: a new epoch of geological time? 369: 835-841. https://doi.org/10.1098/rsta.2010.0339http://ww w. isafreire.pro.br/site/documentos/dessertacao/di ssertacao_roberto_unger.pdf. 